passes, the many banquets, the increased use of limousines, escalators and elevators, less walking, bicycling and stairclimbing, and the continued habit of heavy smoking, all take their toll ${ }^{3}$. The staff gain weight, and develop hypertension, angina pectoris and acute myocardial infarction, some even requiring coronary artery bypass surgery, an operation that was unknown in China until very recently.

TSUNG O. Cheng

Professor of Medicine

The George W ashington University

W ashington, D.C.
1. Novak, K. Cardiovascular disease increasing in developing countries. Nature Med. 4, 989-990 (1998).

2. Tung, C.L. \& Cheng, T.O. in The International Textbook of Cardiology (ed. Cheng, T.O.) 10-14 (Pergamon, New York, 1987).

3. Cheng, T.O. Teenage smoking in China. J. Adolescence (in press)

4. Cheng, T.O. Smoking in China. Br. Med. J. 310, 61 (1995).

\title{
Heat shock proteins, HLA-DR and rheumatoid arthritis
}

In March 1996, Nature Medicine published an article by Auger et al. ${ }^{1}$, accompanied by a News and Views story ${ }^{2}$, making the provocative claim that heat shock protein binding to HLA-DR molecules of certain individuals may profoundly influence susceptibility to rheumatoid arthritis (RA). If true, this would provide a molecular explanation of why Caucasian RA patients are usually HLA-DR4-positive (*0401 or 0404 alleles) or HLA-DR10 positive. Our data do not support this proposal.

The common feature of the disease-associated alleles is that they share a fiveamino-acid motif, called the 'shared epitope', at position $370-74$ of the third hypervariable region: (Q/R)(K/R)RAA. Protective alleles contain a more acidic motif, such as DERAA (ref. 3). Auger, et al. suggested that the stretch of residues constituting the shared epitope specifically recruits a molecular chaperone, HSP73, which targets relevant DRb chains to lysozomes, although it is conceptually difficult to imagine where within the cell the cytosolic/nuclear HSP73 chaperone would interact with luminal DR $\beta 1$ sequence. This work was based upon an earlier observation showing that the bacterial DnaK protein (bacterial homologue of HSP73) could bind

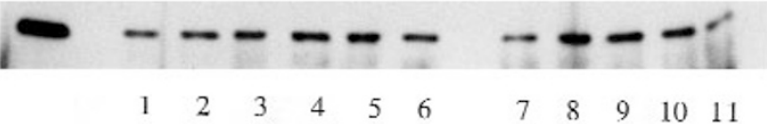

HSP73 co-precipitates nonspecifically with HLA class I and II DR molecules. HLA class I and II proteins were immunoprecipitated from the B lymphoblastoid cell lines SWEIG (lanes 1-3), SAVC (lanes 4-6) and HOR (lanes 7-11) using either the anti-class I monoclonal antibody W6/32 (lanes 3, 6 and 10) or the anti-class II monoclonal antibody B8.12.2. (lanes 1, 2, 4, 5, 8 and 9). Coprecipitation of HSP73 was identified by western blotting with the anti-HSP73 monoclonal antibody SPA-815. The western blot shows that HSP73 co-precipitates with either antibody or with a protein G control (lanes 7 and 11). Immunoprecipitates of B8.12.2. were washed at physiologic salt concentration (lanes 2, 5 and 9) or at $300 \mathrm{mM} \mathrm{NaCl}$ (lanes 1, 4 and 8). The far left track used $20 \mu \mathrm{g}$ total lysate as a positive control for HSP73 binding. Methods: Immunoprecipitation conditions were as described (ref. 1 and J.A. Roudier, pers. comm.). peptide affinity columns comprising the QKRAA conserved tripeptide, HPD recruiting HSP73 (ref. 5) ciation of HSP73. Co-precipitation of HSP73 with HLA-DR was observed in expromiscuously binds protein in certain detergent lysates. This feature reflects its role as a chaperone in recognizing unfolded protein. Immunoprecipitation experiments under more stringent lysis conditions showed that the nonspecific association of HSP73 with all HLA-DR alleles was completely negated in Triton-X100 and CHAPS Iysates in physiologic salt concentrations (not shown).

Our data do not confirm HSP73 DR antigen capture as a molecular mechanism of RA. The initial observation could have been due to minor quantitative variation in non-specific binding, perhaps even post-lysis, or differences in the sensitivity of detection of HSP73 by immunoblotting. HSP73 has dual protein chaperone roles; one is a specific association, targeting protein to Iysosomes, and the other is the ATP-dependent stabilization of hydrophobic regions in extended polypeptide sequences. Partial protein denaturation that may occur in certain detergent Iysates will reveal hydrophobic patches leading to promiscuous binding of HSP73.

The recently demonstrated crystal structure of DRB1*0401 shows the molecule complexed with a collagen type II peptide in which the amino acid at position 71 in the shared epitope participates in both antigen binding and TCR activation $^{6}$. This structure is consistent with existing models for the role of DR in autoimmunity, which deal with specificity of T-cell stimulation.

TINA RICH, ULRIKE GRÜNEBERG \& JOHN TROWSDALE

Department of Pathology, Division of Immunology, University of Cambridge, Tennis Court Road Cambridge, England

Acknowledgments

T.R. was supported by a grant from the Arthritis Research Council; U.G., by a grant from

Boehringer Ingelheim; and J.T., by the Wellcome Foundation

Reply_-Our Nature M edicine article $\mathrm{e}^{1}$ and a follow-up article $e^{4}$ make two points, both challenged by Rich et al. First, the QKRAA amino-acid motif, which, when expressed on HLA-DR, helps the development of rheumatoid arthritis, is also expressed on the Escherichia coli heat shock protein Dnal and helps DnaJ (HSP40) bind partner chaperone DnaK (HSP70). This point is based on the following evidence: The QKRAA motif is expressed in the $\mathrm{N}$-terminal part ( $\mathrm{d}$ domain) of most bacterial DnaJ proteins; peptides con- 
taining the QKRAA motif bind DnaK (allowing its purification from total bacterial proteins). This property is lost if minor changes are introduced in the QKRAA motif; direct binding of Dnal (HSP40) to DnaK (HSP70) is inhibited by QKRAA-containing peptides; and direct binding of DnaJ (HSP40) to DnaK (HSP70) is inhibited by antibody to the QKRAA motif.

The NMR structure of E. coli Dnal (more relevant to the discussion than the structure of a mammalian Dnal, which contains no QKRAA motif) shows that the QKRAA motif is located on an outer alpha helix (as it is on HLA-DRB1*0401) (ref. 7) and does not establish which part of Dnal binds DnaK.

Second, in B cells, HLA-DRB1*0401 (an allele with a QKRAA motif) and HLADRB1*1001 (RRRAA motif) associate with the constitutive HSP70, called HSP73.

This point is based on the following experiments. Immunoprecipitation of HLA-DR from 15 different lymphoblastoid cell lines co-precipitates HSP73 in four of four HLA-DRB1*0401 lines, one of one DRB1*1001 line, and none of the other 10 lines. Immunoprecipitation of HLA-DR after metabolic labelling of Bcell lines homozygous for HLADRB1*0401, 0404, 0101 shows that, in DRB1*0401 cells only, a 70-kDa protein co-precipitates with HLA-DR. After metabolic labelling of $B$ cells and separation of lysosomes, HSP70 co-preci pitates with HLA-DR in lysosomal extracts from DRB1*0401 cells, but not from DRB $1 * 0101$ cells.

The specific association of HLADRB1*0401 or HLA-DRB1*1001 with HSP73 is challenged by Rich et al., because they co-precipitated HSP73 with HLA-DR in three cell lines. One of these, SAVC, expresses DRB1*0401. Using this cell line, we co-precipitated HSP73 with HLA-DR. (The other two cell lines (SWEIG and HOR) do not express DRB1*0401 and we did not test them.) This co-precipitation experiment, done on three cell lines with anti-class I antibody, anti-class II antibody, or protein $G$ or protein A controls, shows positive de tection of HSP73 by immunoblotting. We never observed any HSP73 precipitated by protein $\mathrm{G}$ or protein $\mathrm{A}$ alone, whatever line we worked with. Therefore, we believe that this all-positive blot only indicates overdetection of the anti HSP73 antibody (perhaps resulting from a toolong exposure of the film).

This criticism cannot apply to our pos- itive data because they were also obtained after metabolic labelling, without using anti HSP73 antibody. Still, we are well aware that co-precipitation experiments have one drawback: they do not allow quantitation of the association between HLA-DR and HSP73. Thus, a very weak binding could be represented as a strong one simply by longer exposure of the film. Therefore, in the past two years, we have developed quantitative precipitation assays and direct binding assays to evaluate how well different HLA-DR alleles bind to HSP73.These experiments confirm that HLA-DRB $1 * 0401$ is the best at binding to HSP73, but show that other alleles can bind HSP73 weakly.

Thus, we stand behind our data. They indicate that the QKRAA motif, whether on Dnal or on HLA-DRB1*0401, binds HSP70s. As yet, we do not know if this peculiarity is related to the development of rheumatoid arthritis. Rheumatoid arthritis, in contrast to mouse collagen arthritis, is not (to our knowledge) a disease induced by collagen type II immunization. The fact that HLA-DRB1*0401 may bind collagen II peptides does not indicate that it does so to help the development of rheumatoid arthritis.

ISABelle Auger \& JeAn Roudier Laboratoire d'Immuno Rhumatologie, IN SERM CRI 4U009D

Faculté de Médecine|

13005, Marseille, France

Acknowledgments

Supported by IN SERM, ARP, PHRC 96 and 97 ,

SFR.

1. Auger, I., Escola, J.M., Gorvel, J.P. \& Roudier, J. HLADR4 and HLA-DR10 motifs that carry susceptibility to rheumatoid arthritis bind 70-kD heat shock proteins. Nature Med. 2, 306-310 (1996).

2. Ollier, B. Shock revelations about HLA-DR4-a shortcut to rheumatoid arthritis? Nature M ed. 2, 179-280 (1996).

3. Gregersen, P.K., Silver, J. \& Winchester, R.J. The shared epitope hypothesis - an approach to understanding the molecular genetics of rheumatoid arthritis susceptibility. Arthritis Rheum. 30, 1205-1213 (1987).

4. Auger, I. \& Roudier, J. A function for the QKRAA amino acid motif: mediating binding of dnal to dnaK. J. Clin. Invest. 99, 1818-1822 (1997).

5. Qian, Y.Q., Patel, D., Hartl, F.U. \& McColl, D.J. Nuclear magnetic resonance solution structure of the human Hsp40 (HDJ-1) J-domain. J. Mol. Biol. 260, 224-235 (1996).

6. Dessen, A., Lawrence, C.M., Cupo, S., Zaller, D.M. \& Wiley, D.C. X-ray crystal structure of HLA-DR4 (DRA*0101, DRB1*0401) complexed with a peptide from human collagen II. Immunity 7, 473-481(1997).

7. Szyperski T, Pellechia M, Wall D, Georgopoulos C, Wutrich K. NMR structure determination of the E. coli Dnal molecular chaperone: secondary structure and backbone of the $\mathrm{N}$ terminal region, residues 2-108 containing the highly conserved J domain. Proc. Natl. Acad. Sci. USA 91, 11343-11347 (1994). 